\title{
NATIONAL TAIWAN UNIVERSITY \\ RADIOCARBON MEASUREMENTS II
}

\author{
YUIN-CHI HSU, MUH-CHEN CHOU, YI-CHUAN HSU, \\ SONG-YUN LIN, and SHIH-CHONG LU* \\ Department of Physics, National Taiwan University, \\ Taipei, Taiwan, China
}

The $\mathrm{C}^{1+4}$ dates given below have been obtained by counting $\mathrm{CO}_{2}$ at $2 \mathrm{~atm}$ pressure in a $1 \mathrm{~L}$ proportional counter. Details of procedure are given in our previous list (R., 1970, v. 12, p. 187-192). Radiocarbon dates in this list are based on 95\% of activity of NBS oxalic acid as the modern standard and were calculated using $5570 \mathrm{yr}$ as the half-life of $\mathrm{C}^{14}$. Errors quoted with the dates are standard deviation originating from the statistical nature of radioactive disintegration process. Results obtained during 1970 and 1971 are described here.

\section{ACKNOWLEDGMENT}

This work was supported by the National Council on Science Development.

\section{SAMPLE DESCIRIPTIONS}

I. GEOLOGIC SAMPLES

$$
\text { A. Japan }
$$

\section{NTU-124. Hirogami}

Bogwood from landslide area, Hirogami, Niigata, Japan $\left(37^{\circ} 20^{\prime}\right.$ $\mathrm{N}$ Lat, $139^{\circ} 00^{\prime} \mathrm{E}$ Long), at $0 \mathrm{~m}$ depth. Coll. and subm. 1969 by $\mathrm{S}$. Yamaguchi, Disaster Prevention Inst., Kyoto Univ. Comment (S.Y.): date approximates landslide (Yamaguchi and Lin, 1971).

\section{B. China}

\section{Hwalien series}

Shell and coral of coral reef from Hwalien harbor, Taiwan $\left(23^{\circ} 59^{\prime}\right.$ N Lat, $121^{\circ} 32^{\prime}$ E Long). Coll. 1951 and subm. 1971 by C. C. Lin, Dept. Geol., Natl. Taiwan Univ.

\section{N'TU-159. Hwalien 1}

Coral, at $+3 \mathrm{~m}, 2.5 \mathrm{~m}$ depth.

\section{NTU.166. Hwalien 2}

Shell, at $+3 \mathrm{~m}, 1 \mathrm{~m}$ depth. General Comment (C.C.L.). dates coral reef overlying Milun Formation (Lin, 1969) unconformably and is overlain by the Chara-Melanoides Clay

* Observation Division, Central Weather Bureau, Taipei, Taiwan, China. 
and Sand Member of the Peipin Formation (Lin, 1969). Dates are geologically reasonable.

\section{Hengchun series}

Coral of coral reef from Wangsa village, Hengchun, Taiwan $\left(22^{\circ}\right.$ $03^{\prime} \mathrm{N}$ Lat, $120^{\circ} 45^{\prime}$ E Long), at $+35 \mathrm{~m}$. Coll. and subm. 1971 by C. C. Lin.

NTU-161. Hengchun 1 $>\mathbf{3 0 , 0 0 0}$

Ca. $1 \mathrm{~m}$ depth.

\section{NTU-162. Hengchun 2}

Ca. $1.5 \mathrm{~m}$ depth.

NTU-163. Hengchun 3

Ca. $3 \mathrm{~m}$ depth.

General Comment (C.C.L.): dates are minimum for isolated Pleistocene emerged coral reef, overlain by laterite soil (with gravel), and underlain by Plio-Pleistocene arenaceous limestone of Maopitou Formation (Lin, 1967). Dates are expected.

\section{Taitung series}

Coral and shell of emerged coral reef from Taitung area. Coll. 1960 and subm. 1970 by C. C. Lin.

NTU-150. Taitung 1

$3820 \pm 115$ $121^{\circ} 24^{\prime}$ E Long), at $+20 \mathrm{~m}, 0.5 \mathrm{~m}$ depth.

\section{NTU.151. Taitung 2}

$6132 \pm 184$

Coral from Chiwen village, Taitung, Taiwan $\left(23^{\circ} 08^{\prime} \mathrm{N}\right.$ Lat, $121^{\circ}$ $23^{\prime}$ E Long), at $+35 \mathrm{~m}$, ca. $0.5 \mathrm{~m}$ deptl.

\section{NTU-152. Taitung 3}

$1643 \pm 49$

Coral from Chiten village, Taitung, Taiwan $\left(23^{\circ} 07^{\prime} \mathrm{N}\right.$ Lat, $121^{\circ}$ $22^{\prime}$ E Long), at $+20 \mathrm{~m}$, ca. $0.5 \mathrm{~m}$ depth.

\section{NTU-153. Taitung 4}

$1950 \pm 59$

Coral from Chengkung, Taitung, Taiwan $\left(23^{\circ} 06^{\prime} \mathrm{N}\right.$ Lat, $121^{\circ} 22^{\prime}$ E Long), at ca. $+35 \mathrm{~m}$, ca. $1.5 \mathrm{~m}$ depth.

\section{NTU-154. Taitung 5}

Coral from Chengkung, Taitung, Taiwan $\left(23^{\circ} 06^{\prime} \mathrm{N}\right.$ Lat, $121^{\circ} 22^{\prime}$ E. Long), at ca. $+15 \mathrm{~m}$, ca. $2 \mathrm{~m}$ depth. 
NTU-158. Taitung 6

$9234 \pm 277$

Coral from Fukang valley, Taitung, Taiwan $\left(22^{\circ} 48^{\prime} \mathrm{N}\right.$ $11^{\prime}$ E Long), at $+15 \mathrm{~m}$, ca. $2 \mathrm{~m}$ depth.

\section{NTU-165. Taitung 7}

Shell from Chiwen village, Taitung, Taiwan $\left(23^{\circ} 08^{\prime} \mathrm{N}\right.$ Lat, $121^{\circ}$ $23^{\prime} \mathrm{E}$ Long), at $+35 \mathrm{~m}$, ca. $0.5 \mathrm{~m}$ depth. Comment (C.C.L.): younger than expected.

General Comment (C.C.L.): samples expected to date emerged coral reefs. Except for NTU-165, dates are acceptable.

\section{ARCHAEOLOGIC SAMPLES}

China

\section{Changpin series}

NTU-125. Changpin 4

$>15,000$

Charcoal from LHVI (Sung, 1969) cave, Changpin, Taitung, Taiwan ( $23^{\circ} 24^{\prime} \mathrm{N}$ Lat, $121^{\circ} 25^{\prime} \mathrm{E}$ Long), at $+100 \mathrm{~m}$, ca. $3.35 \mathrm{~m}$ to $3.55 \mathrm{~m}$ depth. Coll. and subm. 1970 by W. H. Sung, Dept. Archaeol. and Anthropol., Natl. 'Taiwan Univ. Comment (W.H.S.): this sample was obtained from geologically and culturally earlier horizon of Changpin culture. Four $\mathrm{C}^{14}$ dates, NTU-69-71 (Hsu et al., 1970) and Y-2638 (Sung, 1969) of the latest phase of this culture range from 5000 to $6000 \mathrm{yr}$ B.P. Thus, present date not only agrees with above-mentioned dates but also with estimate of W. H. Sung (1969), that earlier phase of Changpin culture must go back to Pleistocene.

III. GEOPHYSICAL SAMPLES

\section{$C^{t 4}$ in Atmospheric Carbon Dioxide}

\section{Atmospheric radiocarbon activity series, Taipei}

$\mathrm{C}^{14}$ content in ground level atmospheric $\mathrm{CO}_{2}$ is monitored monthly at Taipei, Taiwan $\left(25^{\circ} 02^{\prime} \mathrm{N}\right.$ Lat, $121^{\circ} 32^{\prime} \mathrm{E}$ Long). The following list contains exposure time at $\mathrm{NaOH}$ solutions to air and per cent increase of $\mathrm{C}^{1-4}$ above $95 \%$ NBS oxalic acid. Data are graphed in Fig. 1. The statistical error is less than $1 \%$.

\begin{tabular}{|c|c|c|c|}
\hline Sample no. & Exposure time & & $\delta \mathrm{C}^{1 \pm}, \%$ \\
\hline NTU-113 & 15 Jan. -20 Jan. 19 & 1968 & +56.6 \\
\hline NTU-114 & 15 Feb. -21 Feb. 19 & 1968 & +59.5 \\
\hline NTU-115 & 15 Mar. - 20 Mar. 19 & 1968 & +61.0 \\
\hline NTU-116 & 15 Apr. -21 Apr. 19 & 1968 & +56.0 \\
\hline NTU-117 & 18 May - 23 May 19 & 1968 & +49.9 \\
\hline NTU-118 & 16 June -22 June 19 & 1968 & +55.5 \\
\hline NTU-119 & 15 July & 1968 & +55.9 \\
\hline
\end{tabular}




\begin{tabular}{|c|c|c|}
\hline Sample no. & Exposure time & $\delta \mathrm{C}^{14}, \%$ \\
\hline NTU-120 & 20 Sept. -25 Sept. 1968 & +55.6 \\
\hline NTU-121 & 18 Oct. -22 Oct. 1968 & +53.5 \\
\hline NTU-122 & 15 Nov. -20 Nov. 1968 & +55.0 \\
\hline N'IU-123 & 17 Dec. - 22 Dec. 1968 & +54.7 \\
\hline NTU-126 & 15 Jan. - 21 Jan. 1969 & +51.2 \\
\hline NTU-127 & 15 Feb. - 21 Feb. 1969 & +47.2 \\
\hline NTU-128 & 16 Mar. - 21 Mar. 1969 & +52.7 \\
\hline NTU-129 & 16 Apr. - 21 Apr. 1969 & +43.7 \\
\hline NTU-130 & 16 May - 21 May 1969 & +67.7 \\
\hline NTU-131 & 17 June - 21 June 1969 & +54.2 \\
\hline NTU-132 & 15 Aug. - 21 Aug. 1969 & +49.4 \\
\hline N'IU-133 & 15 Sept. - 21 Sept. 1969 & +50.7 \\
\hline NTU-134 & 15 Oct. -21 Oct. 1969 & +40.0 \\
\hline NTU-135 & 15 Nov. - 20 Nov. 1969 & +54.1 \\
\hline NTU-136 & 15 Dec. -20 Dec. 1969 & +74.7 \\
\hline NTU-137 & 15 Jan. -20 Jan. 1970 & +52.4 \\
\hline N'IU-138 & 14 Feb. -18 Feb. 1970 & +55.7 \\
\hline NTU-139 & 15 Mar. - 21 Mar. 1970 & +55.9 \\
\hline NTU-140 & 15 Apr. - 20 Apr. 1970 & +56.4 \\
\hline NTU-141 & 15 May - 21 May 1970 & +55.7 \\
\hline NTU-142 & 15 June -21 June 1970 & +56.2 \\
\hline NTU-143 & 15 July -20 July 1970 & +55.0 \\
\hline NTU-144 & 15 Aug. - 20 Aug. 1970 & +45.7 \\
\hline NTU-145 & 14. Sept. - 21 Sept. 1970 & +52.6 \\
\hline NTU-146 & 14 Oct. -20 Oct. 1970 & +49.8 \\
\hline NTU-147 & 15 Nov. - 20 Nov. 1970 & +44.7 \\
\hline NTU-I 48 & 14 Dec. -20 Dec. 1970 & +48.9 \\
\hline
\end{tabular}

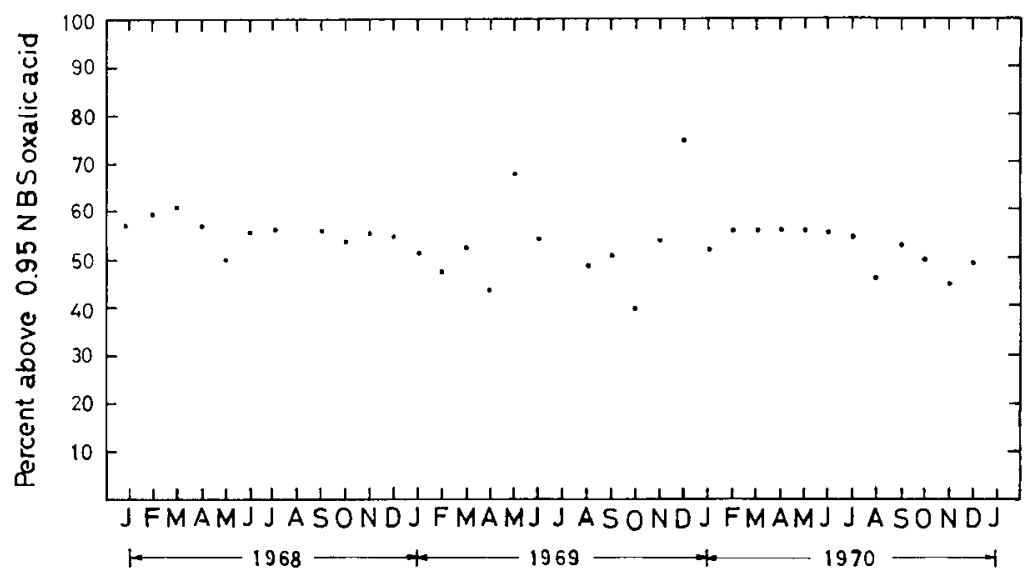

Fig. 1. $\mathrm{C}^{14}$ enrichment over NBS standard of atmospheric $\mathrm{CO}_{2}$ during 1968 to 1970 at Taipei, Taiwan $25^{\circ} 02^{\prime} \mathrm{N}$ Lat, $121^{\circ} 31^{\prime}$ E Long). 
REFERFNCES

Hsu, Y. C. et al,, 1970, National Taiwan University radiocarbon measurcments I: Radiocarbon, v. 12, p. 187-192.

Lin, C. C., 1967, Quaternary of Taiwan VIII, Quaternary system of the Hengchun Peninsula: Rept., Natl. Council on Sci. Development.

1969, Holocene geology of Taiwan: Acta Geol. Taiwanica, no. 13, Dec., p. 83-126.

Sung, W. H., 1969, Changpin: a newly discovered Preceramic culture from the Ag. glomerate Caves on cast coast of Taiwan: Newsletter Chinese Ethnol., no. 9.

Yamaguchi, S. and Lin, T. H., 197l, The clecision of moving cycle of landslide in past time by the age measurement of bogwood obtained from landslide area: Lindslide, v. 7 , p. $1-6$. 